\title{
An Analysis of Risk Factors, Timing of Complications and Readmission after Pancreaticoduodenectomy
}

\author{
John V Gahagan*, Mark H Hanna, Zhobin Moghadamyeghaneh, Reza Fazl Alizadeh, Adrienne \\ Nguyen, Maki Yamamoto, Michael J Stamos, Aram N Demirjian and David K Imagawa \\ Department of Surgery, University of California, Irvine School of Medicine, Irvine, California, USA
}

Received: October 04, 2017; Accepted: February 14, 2018; Published: March 05, 2018

*Corresponding author: John V Gahagan, Department of Surgery, University of California, Irvine School of Medicine, Irvine, California, USA, Email: gahaganj@uci.edu; Fax: (714) 456-7207

\begin{abstract}
Objective: Postoperative complications after pancreatico duodenectomy (PD) have been associated with an increased risk of readmission, yet the timing of these complications is unclear.

Methods: The NSQIP database was reviewed to identify patients who underwent PD in 2012. A multivariate analysis assessed postoperative outcomes, timing of complications and impact on readmission.

Results: 3,277 patients underwent PD: $48 \%$ experienced a postoperative complication and $16 \%$ were readmitted. The readmission rate in those that experienced a postoperative complication was $23 \%$. After a postoperative complication, the readmission rate was $15 \%$ for pre-discharge compared with $70 \%$ for post-discharge complications $(\mathrm{P}<0.05)$. Predictors of readmission included: hypertension $(\mathrm{RR}=1.33, P<0.05)$, re-operation $(\mathrm{RR}=1.85, P<0.05)$ dependent functional status $(\mathrm{RR}=2.6, P<0.05)$, duodenal neoplasm $(\mathrm{RR}=8.81, P<0.05)$, cystic disease $(\mathrm{RR}=17.07, P<0.05)$ and the occurrence of post-discharge complications $(\mathrm{RR}=21.59, P<0.05)$. $89 \%$ of all complications occurred pre-discharge and $11 \%$ occurred postdischarge. Among post-discharge complications, deep space infection was the most common to occur (3.8\%) and resulted in a readmission rate of $91 \%$.
\end{abstract}

Conclusions: Pre-discharge complications were more common but post-discharge complications had a 20 -fold increased risk of readmission.

Keywords: Readmission; Pancreaticoduodenectomy; Whipple; NSQIP; Morbidity

\section{Introduction}

The first successful pancreaticoduodenectomy (PD) was performed and reported by Whipple, Parsons and Mullins in 1935[1]. The authors reported a case series of three patients who underwent a two stage procedure for the treatment of ampullary carcinoma. A one-stage PD was later reported by Trimble in 1941. During the early stages of this procedure, morbidity and mortality were exceedingly high, up to $50 \%$ and $30 \%$, respectively [2-4].

Beginning in the 1970s, peri-operative mortality began to decline $[3,4]$. Contemporary reports of peri-operative mortality after PD in high-volume centers are approximately 2\% [4-6]. This improvement has resulted from a variety of advancements in surgical technique, critical care, and management of postoperative complications. Although mortality has improved dramatically, morbidity has remained relatively high, at around $40-50 \%[4,6,7]$.

Because PD continues to be a high-risk procedure, the overall 30-day readmission rate is approximately $15-25 \%$. [69]. Readmission rates of up to $38 \%$ have been reported during the 90 day peri-operative period [10]. Previous studies have shown an association between post-operative complications and readmission after PD $[7,9]$. However, it is currently unclear which complications put patients at highest risk for readmission and how the timing of complications affects this risk.

The objectives of this study were threefold. First, we sought to identify patient characteristics and hospital factors associated with readmission in a large national cohort. Second, we identified patients who experienced a complication after PD to examine how the readmission rate is affected by the occurrence of specific post-operative complications. Third, we characterized complications as occurring before discharge or after discharge to examine the timing of specific complications and to evaluate how the timing of these complications impacts the readmission rate.

\section{Methods}

Database: The American College of Surgeons National Surgical Quality Improvement Program (ACS NSQIP) database is the first national validated, outcomes-based program designed for the purpose of improving surgical quality of care. In 2012, the ACS NSQIP database collected data from 374 participating sites on more than 300 variables, including preoperative risk factors, 
intraoperative variables, and 30-day postoperative mortality and morbidity outcomes for patients undergoing major surgical procedures in both the inpatient and outpatient setting. A trained surgical clinical reviewer is used at participating hospitals to standardize data capture and ensure reliability.

The ACS NSQIP database and the hospitals participating in the ACS NSQIP are the source of the data used herein. They have not verified and are not responsible for the statistical validity of the data analysis or the conclusions derived by the authors. Approval for the use of patient level data analyzed in this study was obtained from the Institutional Review Board of the University of California Irvine Medical Center and from the ACS NSQIP.

Participant Selection: A retrospective review of the ACS NSQIP Participant User File (PUF) database was performed. Patients who underwent a PD in 2012 were identified using Current Procedural Terminology (CPT) codes: 48150, 48152, 48153, and 48154. The publically available ACS NSQIP PUFs began reporting readmission data in 2011 and capture readmission to any hospital occurring within 30 days of the index procedure. Furthermore, the ACS NSQIP captures both inpatient and outpatient postoperative complications within that same 30 day period. Patients under the age of 18 years (inherent NSQIP exclusion), those with incomplete readmission data, or those that died within 30 days of the operation were excluded from analysis.

Demographics and outcomes variables: Analyzed variables included: patient demographics (age, sex, race), comorbid conditions (hypertension, dialysis, dyspnea, chronic obstructive pulmonary disease, smoking, diabetes mellitus, congestive heart failure, ascites, steroid use), body mass index (BMI) (derived from height and weight), American Society of Anesthesiologists (ASA) classification, functional status, operative factors (indication, operation time, wound classification, return to the operating room), and hospital factors (length of stay, type of admission, discharge destination).

Post-operative complications and short-term operative outcomes analyzed included: superficial surgical site infection
(SSI), deep SSI, organ space SSI, wound disruption, pneumonia, unplanned intubation, on ventilator $>48$ hours, deep vein thrombosis, pulmonary embolism, acute renal failure, progressive renal insufficiency, urinary tract infection, stroke, cardiac arrest, myocardial infarction, bleeding requiring transfusion, sepsis, and septic shock.

Post-operative complications were then classified as occurring either before or after discharge. Within the ACS NSQIP database, the time (in days) to the onset of a particular complication is tracked. If the time to a complication was less than or equal to the time to discharge, the complication was deemed to have occurred prior to discharge. Patients were classified as those who experienced no complication, those who experienced a complication prior to discharge, and those who experienced a complication after discharge. Readmission rates were then calculated for each group.

Statistical analysis: Statistical analysis was performed with SPSS software, version 22 (IBM Corp, Armonk, NY). All of the factors were analyzed using univariate logistic regression. Forward stepwise multivariable regression models including all of the factors approaching significance $(p<0.1)$ on univariate analysis were used to identify factors associated with readmission. The odds ratios generated through regression were adjusted to risk ratios (RR) 95\% confidence intervals (CI) given the high frequency of readmissions [11]. Statistical significance was declared if $p<0.05$.

\section{Results}

A total of 3,722 patients who underwent a PD in 2012 were identified within the ACS NSQIP database. The overall readmission rate was $16.2 \%(n=532)$. Overall, $48 \%$ of patients $(n=1585)$ experienced a post-operative complication. The readmission rate among patients who experience a post-operative complication was $23 \%$. The readmission rate among patients who did not experience a post-operative complication was $10 \%$

Patient demographics, comparing those readmitted to those not readmitted, are shown in table 1.

\begin{tabular}{|c|c|c|c|c|}
\hline Patient Characteristics & $\begin{array}{c}\text { Readmitted } \\
(n=532)\end{array}$ & $\begin{array}{l}\text { Not readmitted } \\
\quad(n=2745)\end{array}$ & $\begin{array}{c}\text { Total Cohort } \\
(n=3277)\end{array}$ & p-value \\
\hline \multicolumn{5}{|l|}{ Age } \\
\hline Mean, year* & $64 \pm 12$ & $65 \pm 12$ & $65 \pm 12$ & 0.04 \\
\hline Median, year & 64 & 66 & 66 & \\
\hline Female gender & $259(48.7 \%)$ & $1273(46.4 \%)$ & 1532(46.8\%) & 0.32 \\
\hline Emergent admission & $3(0.6 \%)$ & $17(0.6 \%)$ & $20(0.6 \%)$ & 0.88 \\
\hline \multicolumn{5}{|l|}{ Functional Status } \\
\hline Independent & $522(98.1 \%)$ & $2717(99 \%)$ & $3239(98 \%)$ & 0.12 \\
\hline Dependent & $10(1.9 \%)$ & $26(1 \%)$ & $33(1 \%)$ & 0.12 \\
\hline
\end{tabular}




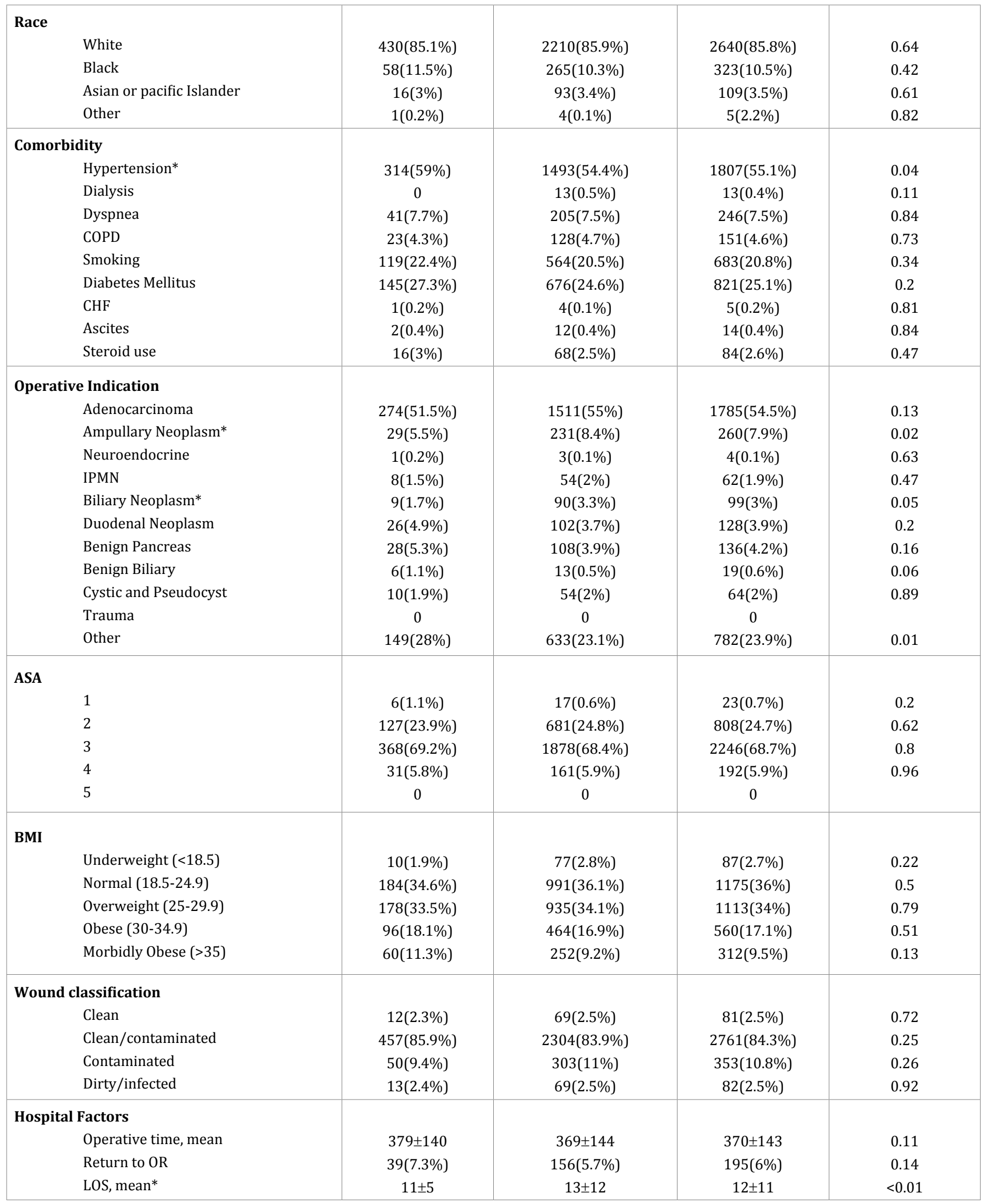




\begin{tabular}{|l}
\hline Discharge Destination \\
Skilled Care (not home) \\
Unskilled Facility (not home) \\
Facility (Home) \\
Home* \\
Separate Acute Care \\
Rehab \\
Expired \\
Unknown
\end{tabular}

$51(9.6 \%)$
0
$2(0.4 \%)$
$455(85.5 \%)$
$3(0.6 \%)$
$20(3.8 \%)$
$1(0.2 \%)$
0

* Indicated statistically significant difference between readmitted patients and not readmitted patients

Overall, there was no statistical difference between the two groups with respect to sex, race, comorbid conditions (except hypertension), BMI, ASA classification, functional status, operative time, wound classification, length of stay, and discharge destination. The readmitted group had a slightly lower mean age (64 years vs. 65 years, $p<0.05$ ), as well as a higher rate of hypertension ( $59 \%$ vs $54 \%, p<0.05$ ), compared to the nonreadmitted group. With respect to operative indications, the readmitted group had lower rates of ampullary (5.5\% vs $8.4 \%$, $p<0.05)$ and biliary ( $1.7 \%$ vs $3.3 \%, p<0.05)$ neoplasm's, compared to the non-readmitted group.In looking at hospital factors, the readmitted group had, on average, a shorter length of stay (11 days vs. 13 days, $p<0.05$ ) and a higher rate of discharge to home $(85 \%$ vs $82 \%, p<0.05)$ rather than to another living facility.
A forward stepwise multivariable regression analysis for all of the factors approaching significance $(p<0.1)$ on univariate analysis was performed to identify factors associated with readmission, which is shown in table 2 . Having a cystic neoplasm, duodenal neoplasm, or an unspecified tumor type was associated with readmission, with a relative risk of 17 (95\%CI 1.06274.24), 8.8(95\%CI 2.03-38.18) and 2.8 (95\%CI 1.17-6.83), respectively. Having a dependent functional status as well as having hypertension were associated with readmission, with a relative risk of 2.6 (95\%CI 1.04-6.50) and 1.3 95\%CI 1.03-1.71), respectively. Return to the operating room was also associated with readmission, with a relative risk of 1.85 (95\%CI 1.12-3.05). In contrast, a longer length of stay was associated with decreased rate of readmission, with a relative risk of 0.97 (95\%CI 0.95-0.99). This association with length of stay may be due to an inherent bias within the database, which is discussed below.

\begin{tabular}{|c|c|c|c|}
\hline Table 2: Relative risk of readmission using multivariable analysis for all of the factors approaching significance $(p<0.1)$ on univariate analysis \\
\hline Patient Characteristics & RR & $\mathbf{9 5 \%}$ CI & P value \\
\hline Cystic and Pseudocyst & 17.07 & $1.06-274.24$ & $<0.05$ \\
\hline Duodenal Neoplasm & 8.81 & $2.03-38.18$ & $<0.05$ \\
\hline Other tumor type & 2.83 & $1.17-6.83$ & $<0.05$ \\
\hline Partially or Totally dependent & 2.6 & $1.04-6.50$ & $<0.05$ \\
\hline Hypertension & 1.33 & $1.03-1.71$ & $<0.05$ \\
\hline Return to operating room & 1.85 & $1.12-3.05$ & $<0.05$ \\
\hline Length of stay, mean & 0.97 & $0.95-0.99$ & $<0.05$ \\
\hline
\end{tabular}

Overall, 48\% of those who underwent a PD (1585 patients) experienced at least one complication during the 30-day perioperative period. Complications were then classified as occurring before discharge or after discharge. Of all the patients who underwent a PD, 42.8\% ( $\mathrm{n}=1403)$ experienced at least one complication before discharge and $11 \%(n=361)$ experienced at least one complication after discharge. Additionally, 4.8\% $(n=179)$ experienced a complication before discharge as well as after discharge.

The most common complication was bleeding requiring transfusion, experienced by $26 \%$ of patients. All of the bleeding complications occurred before discharge, which is an artifact of the database. Bleeding, requiring blood transfusion is tracked from the time of surgery up to 72 hours post-operatively. The second most common complication was organ space SSI, occurring in $8.3 \%$ of patients before discharge and $3.8 \%$ of patients after discharge. Organ space SSI was also the most common complication to occur after discharge. Readmission rate associated with each complication, depending on whether the complication occurred before discharge or after discharge were then calculated. Bleeding, which was the most common complication overall, had a readmission rate of $16 \%$. Organ space SSI had a readmission rate of $8.5 \%$ when it occurred before discharge and $91.3 \%$ when it occurred after discharge. The readmission rates for complications that occurred before discharge ranged from $0-19.4 \%$. The readmission rates for complications that occurred after discharge ranged from 42.1$100 \%$ (Table 3). 
Table 3: Readmission rates for complications occurring before or after discharge

\begin{tabular}{|c|c|c|c|c|c|}
\hline & $\begin{array}{c}\text { Overall } \\
\text { Complication } \\
\text { Rate N (\%) }\end{array}$ & $\begin{array}{c}\text { Pre-discharge } \\
\text { complication rate }\end{array}$ & $\begin{array}{l}\text { Post-discharge } \\
\text { complication rate }\end{array}$ & $\begin{array}{l}\text { Readmission rate } \\
\text { when occurring } \\
\text { before discharge }\end{array}$ & $\begin{array}{c}\text { Readmission rate } \\
\text { when occurring after } \\
\text { discharge }\end{array}$ \\
\hline Superficial SSI & $335(10.2 \%)$ & $228(6.9 \%)$ & $107(3.3 \%)$ & $14.5 \%$ & $42.1 \%$ \\
\hline Deep SSI & $98(3 \%)$ & $59(1.8 \%)$ & $39(1.2 \%)$ & $18.6 \%$ & $79.5 \%$ \\
\hline Organ/Space SSI & $398(12.1 \%)$ & $272(8.3 \%)$ & $39(1.2 \%)$ & $8.5 \%$ & $91.3 \%$ \\
\hline Wound disruption & $59(1.8 \%)$ & $40(1.2 \%)$ & $19(0.6 \%)$ & $17.5 \%$ & $84.2 \%$ \\
\hline Urinary Tract Infection & $160(4.9 \%)$ & $136(4.15 \%)$ & $24(0.7 \%)$ & $14.7 \%$ & $62.5 \%$ \\
\hline DVT requiring treatment & $91(2.8 \%)$ & $67(2 \%)$ & $24(0.7 \%)$ & $14.9 \%$ & $66.7 \%$ \\
\hline Pulmonary embolus & $34(1 \%)$ & $25(0.7 \%)$ & $9(0.3 \%)$ & $20.0 \%$ & $77.8 \%$ \\
\hline Pneumonia & $137(4.2 \%)$ & $120(3.6 \%)$ & $17(0.5 \%)$ & $10.0 \%$ & $100 \%$ \\
\hline $\begin{array}{l}\text { Bleeding requiring } \\
\text { transfusion }\end{array}$ & $854(26 \%)$ & $854(26 \%)$ & 0 & $16.0 \%$ & $\mathrm{~N} / \mathrm{A}$ \\
\hline Unplanned intubation & $153(4.7 \%)$ & $135(4.1 \%)$ & $18(0.5 \%)$ & $7.4 \%$ & $100 \%$ \\
\hline Ventilator $>48$ hour & $133(4 \%)$ & $124(3.7 \%)$ & $9(0.3 \%)$ & $5.6 \%$ & $100 \%$ \\
\hline $\begin{array}{l}\text { Progressive renal } \\
\text { insufficiency }\end{array}$ & $34(1 \%)$ & $29(0.8 \%)$ & $5(0.2 \%)$ & $13.8 \%$ & $100 \%$ \\
\hline Acute renal failure & $42(1.3 \%)$ & $38(1.1 \%)$ & $4(0.1 \%)$ & $0 \%$ & $75 \%$ \\
\hline Cerebrovascular accident & $15(0.5 \%)$ & $10(0.3 \%)$ & $5(0.2 \%)$ & $10 \%$ & $80 \%$ \\
\hline Cardiac Arrest requiring CPR & $38(1.2 \%)$ & $35(1 \%)$ & $3(0.1 \%)$ & $0 \%$ & $100 \%$ \\
\hline MI & $39(1.2 \%)$ & $31(0.9 \%)$ & $8(0.2 \%)$ & $19.4 \%$ & $75 \%$ \\
\hline Sepsis & $295(9 \%)$ & $221(6.7 \%)$ & $74(2.3 \%)$ & $11.8 \%$ & $95.5 \%$ \\
\hline Septic Shock & $137(4.2 \%)$ & $122(3.7 \%)$ & $15(0.5 \%)$ & $2.5 \%$ & $100 \%$ \\
\hline
\end{tabular}

Overall, if a patient experienced any complication before discharge, the readmission rate was $15 \%$. If a patient experienced any complication after discharge, the readmission rate was $70 \%$. This difference was statistically significant $(p<0.05)$. On multivariate analysis, a complication after discharge had a relative risk of readmission of 21.59 (95\%CI 16.48-28.28) (Table 4).

Table 4: Relative risk of readmission based on the timing of the complication using a multivariable analysis

\begin{tabular}{|c|c|c|c|}
\hline Patient Characteristics & RR & $\mathbf{9 5 \%}$ CI & P value \\
\hline $\begin{array}{c}\text { Pre-discharge Occurrence } \\
\text { of Complications }\end{array}$ & 1.1 & $0.86-1.41$ & 0.42 \\
\hline $\begin{array}{c}\text { Post-discharge Occurrence } \\
\text { of Complications }\end{array}$ & 21.59 & $16.48-28.28$ & $<0.05$ \\
\hline
\end{tabular}

\section{Discussion}

$\mathrm{PD}$ is a complex operation with a high inherent risk for complication and readmission, but studies examining the reasons for readmission after PD are sparse and most are based on single institution data [7, 8, 10, 12-15]. None of these studies have examined how the timing of complications might impact readmission rates. The present study highlights a few important points. First, at a national level with contemporary data, PD remains a high-morbidity procedure, with a substantial readmission rate: up to $23 \%$ in patients who developed a postoperative complication compared to $10 \%$ in those who did not. Complications that occur after discharge are associated with a significantly higher readmission rate compared to complications that occur prior to discharge. Furthermore, we found that the most frequent complications after PD were bleeding before discharge and infectious complications after discharge.

In our results, there did not appear to be clinically relevant demographic differences between patients who were readmitted and those who were not readmitted to provide an explanation for the need for readmission. Although we found a statistical difference in age and the rate of hypertension, these differences are likely trivial and not clinically significant. In contrast, tumor characteristics were associated with readmission. Patients who underwent PD for cystic or pseudo-cystic lesions as well as for malignant tumors in the duodenum had significantly higher rates of readmission. It is possible that this is a function of the consistency of the pancreas and the size of the pancreatic duct which are both risk factors for pancreatic fistula. This information is not available in the 2012 NSQIP database but will now be available with the specific pancreatic data parameters which were incorporated in 2015. 
We found an overall complication rate of $48 \%$, which is consistent with previous single institution studies reporting rates of $30-58 \%[4,8,9,16]$. Although post-operative complications have previously been associated with readmission [7-9, 14], our results show that only complications that occur after discharge are associated with readmission. We found no statistically significant difference in the risk of readmission between those who experienced a complication before discharge and those that did not experience any complication. In contrast, patients who experienced a complication after discharge had a significantly increased risk of readmission compared to those that did not experience a complication. While it may seem intuitive that having a complication after discharge puts patients at higher risk for readmission, it is interesting that having a complication before discharge does not appear to increase the readmission risk. These data suggest that even though we might expect patients who experience a complication before discharge to be more debilitated or sick, addressing a complication during the index hospitalization seems to return patients to their "baseline" state of health as if they had experienced no complication. Similarly, when complications occur after discharge, patients are not likely to be in a position to have that particular complication identified and treated immediately, as might occur if they were still in an acute care setting. Therefore, when the complication is recognized, it may be at a more advanced stage requiring more intensive management and possibly readmission.

Although our data show that patients who experience a complication after discharge have a significantly higher rate of readmission, it is difficult to delineate the specific reasons for readmission. For some complications that occur after discharge, such as septic shock or unplanned intubation, a readmission is understandable and expected. However, for other complications that occur after discharge, such as a superficial SSI or UTI, one might expect that these could be treated on an outpatient basis without the need for a readmission. Yet there were substantial rates of readmission associated with superficial SSI and UTI that occurred after discharge, $42 \%$ and $62 \%$, respectively. As with all statistical analyses, these associations do not imply causation, and reasons for readmission are not well captured in NSQIP. Moreover, PD-specific complications, such as delayed gastric emptying and pancreatic leak are also not captured within NSQIP. Also, dependent functional status was associated with readmission. Therefore, other factors that could not be examined in this study may have contributed to the readmissions, and these seemingly benign complications may simply be a marker of overall illness rather than the impetus for readmission. In other words, these complications may have been discovered incidentally upon readmission for another reason. These findings help explain why, despite a significant research effort into this area, prediction of readmissions after surgery has proved to be so challenging. The clinical reality is that the reasons for most readmissions after surgery are complex and nuanced, and probably influenced by many poorly captured socioeconomic factors.

In our study, a shorter post-operative hospital stay was associated with readmission. This finding is in contrast to multiple previous studies, which have demonstrated that longer hospital stay is associated with both early and late readmission after PD [7-9, 13, 15]. Within NSQIP, readmission is tracked up to 30 days from the day of the principle operation rather than from the day of discharge. Consequently, patients who have a longer hospital stay have fewer days for which readmission will be recorded. Therefore, we believe this study's result could be an artifact of the NSQIP database. Nevertheless, our result highlights a struggle for many surgeons: decreasing hospital length of stay while simultaneously minimizing readmissions. The Centers for Medicare \& Medicaid Services (CMS) introduced the Prospective Payment System (PPS) in 1983, which provides a fixed reimbursement for a particular admission and diagnosisrelated group (DRG). After the implementation of PPS, there was a dramatic decrease in average length of stay [17], with a concomitant increase in the rate of readmission [18]. To incentivize a reduction in readmission, CMS recently introduced the Hospital Readmissions Reduction Program (HRRP) as well as the Bundled Payments for Care Improvement (BPCI). The full effects of these programs are yet to be seen. Nevertheless, because of the high morbidity of PD, surgeons likely will continue to struggle with finding an appropriate balance between length of stay and readmission. Based on our results, we believe that initiatives directed at lowering rates of post-discharge complications may also help reduce readmissions.

There are a few limitations to this study. The ACS NSQIP database is limited to 30-day postoperative morbidity and mortality; therefore, any complications or readmissions occurring after this time period are not captured. Also, the post-operative complications tracked are generic and not PDspecific, such as delayed gastric emptying, pancreatic leak, or marginal ulceration. Readmission is defined as within 30 days from surgical procedure, not from hospital discharge. Therefore, patients who have a longer length of stay have fewer days in which a readmission will be captured, leading to bias in the analysis. Finally, although ACS-NSQIP extensively tracks patient comorbidities and many potential postoperative complications, it lacks many social and economic factors (insurance status, income, education, etc.) that may affect the risk or readmission. Despite these limitations, this study provides an overview of the types of complications that occur after PD and how those complications influence readmission.

\section{Conclusions}

Pancreaticoduodenectomy continues to be a high-risk procedure with high rates of post-operative complications and readmission. The occurrence of a complication before discharge is not associated with an increased rate of readmission. While numerous factors were found to influence the risk of readmission in our study, the single most influential factor was the occurrence of a complication after discharge (readmission rate of $70 \%$ and a relative risk of readmission of 21). Initiatives directed at lowering rates of post-discharge complications and identifying high risk patients that may benefit from closer postoperative surveillance may help reduce the rate of readmissions after PD. 


\section{References}

1. Whipple AO, Parsons WB, Mullins CR. Treatment of Carcinoma of the Ampulla of Vater. Ann Surg. 1935;102(4):763-779.

2. Shapiro TM. Adenocarcinoma of the pancreas: a statistical analysis of biliary bypass vs Whipple resection in good risk patients. Ann Surg. 1975;182(6):715-721.

3. Crist DW, Sitzmann JV, Cameron JL. Improved hospital morbidity, mortality, and survival after the Whipple procedure. Ann Surg. 1987;206(3):358-365.

4. Winter JM, Cameron JL, Campbell KA, Arnold MA, Chang DC, Coleman J, et al. 1423 pancreaticoduodenectomies for pancreatic cancer: A single-institution experience. J Gastrointest Surg. 2006;10(9):11991210

5. Balcom JH, Rattner DW, Warshaw AL, Chang Y, Fernandez-del Castillo C. Ten-year experience with 733 pancreatic resections: changing indications, older patients, and decreasing length of hospitalization. Arch Surg. 2001;136(4):391-398.

6. Lee GC, Fong ZV, Ferrone CR, Thayer SP, Warshaw AL, Lillemoe KD, et al. High performing whipple patients: factors associated with short length of stay after open pancreaticoduodenectomy. J Gastrointest Surg. 2014;18(10):1760-1769. doi: 10.1007/s11605-014-2604-3

7. Emick DM, Riall TS, Cameron JL, Jordan M, Keith D, JoAnn Coleman, et al. Hospital readmission after pancreaticoduodenectomy. J Gastrointest Surg. 2006;10(9):1243-1253. doi: 10.1016/j.gassur.2006.08.016

8. Grewal SS, McClaine RJ, Schmulewitz N, Alzahrani MA, Hanseman DJ, Sussman JJ, et al. Factors associated with recidivism following pancreaticoduodenectomy. HPB (Oxford). 2011;13(12):869-875. doi: 10.1111/j.1477-2574.2011.00377.x

9. Schneider EB, Canner JK, Gani F, Kim Y, Ejaz A, Spolverato G, et al. Early versus late hospital readmission after pancreaticoduodenectomy. J Surg Res. 2015;196(1):74-81. doi: 10.1016/j.jss.2015.02.043
10. Van Geenen RC, van Gulik TM, Busch OR, de Wit LT, Obertop H, Gouma DJ. Readmissions after pancreatoduodenectomy. Br J Surg. 2001;88(11):1467-1471. doi: 10.1046/j.0007-1323.2001.01900.x

11.Zhang J, Yu KF. What's the relative risk? A method of correcting the odds ratio in cohort studies of common outcomes. JAMA. 1998;280(19):1690-1691.

12.Zhu ZY, He JK, Wang YF, Liang X, Yu H, Wang XF, et al. Multivariable analysis of factors associated with hospital readmission following pancreaticoduodenectomy for malignant diseases. Chin Med J. 2011;124(7):1022-1025.

13. Sadot E, Brennan MF, Lee SY, Allen PJ, Gönen M, Groeger JS, et al. Readmission after pancreatic resection: causes and causality pattern. Ann Surg Oncol. 2014;21(13):4342-4350. doi: 10.1245/s10434-0143841-0

14.Ahmad SA, Edwards MJ, Sutton JM, Grewal SS, Hanseman DJ, Maithel SK, et al. Factors influencing readmission after pancreaticoduodenectomy: a multi-institutional study of 1302 patients. Ann Surg. 2012;256(3):529-537.

15. Yermilov I, Bentrem D, Sekeris E, Jain S, Maggard MA, Ko CY, et al. Readmissions following pancreaticoduodenectomy for pancreas cancer: a population-based appraisal. Ann Surg Oncol. 2009;16(3):554-561. doi: 10.1245/s10434-008-0178-6

16. Schmidt CM, Powell ES, Yiannoutsos CT, Howard TJ, Wiebke EA, Wiesenauer CA, et al. Pancreaticoduodenectomy: a 20-year experience in 516 patients. Arch Surg. 2004;139(7):718-725;discussion 725727. doi: 10.1001/archsurg.139.7.718

17. DeFrances CJ, Hall MJ. 2005 National Hospital Discharge Survey. Adv Data. 2007;1-19.

18. DesHarnais S, Hogan AJ, McMahon LF, Fleming S. Changes in rates of unscheduled hospital readmissions and changes in efficiency following the introduction of the Medicare prospective payment system. An analysis using risk-adjusted data. Eval Health Prof. 1991;14(2):228252. 